\title{
Wer darf gegen kantonale Entscheide in Sachen TARMED-Taxpunktwerte Beschwerde erheben?
}

\author{
S. Schneider
}

Korrespondenz:

Sandra Schneider Bundesamt für Sozialversicherung Kranken- und Unfallversicherung Tarife und Leistungserbringer Effingerstrasse 20 CH-3003 Bern
Am 30. September 2002 hat der Bundesrat die Rahmenverträge zwischen Kostenträgern und Leistungserbringern zum Tarif für ärztliche Leistungen in der Krankenversicherung genehmigt. In den Verträgen einigten sich die Tarifpartner einerseits auf die gesamtschweizerisch einheitliche Tarifstruktur für ärztliche Leistungen TARMED in der Version 1.1, andererseits auf Modalitäten zum Tarif (wie Beitritt und Kündigung). Nicht enthalten ist für die obligatorische Krankenpflegeversicherung der anwendbare Taxpunktwert. Dieser ist nach dem Willen der Vertragspartner auf kantonaler oder regionaler Ebene auszuhandeln bzw. bei Nichteinigung von der zuständigen Kantonsregierung festzulegen [1]. Während gegen die Genehmigung der TARMED-Rahmenverträge und die Festlegung der Version 1.1 des TARMED als gesamtschweizerische einheitliche Tarifstruktur durch den Bundesrat keine Beschwerdemöglichkeit besteht, kann gegen Entscheide auf kantonaler Ebene zu den Taxpunktwerten beim Bundesrat Beschwerde erhoben werden [2]. Mit Blick auf das KVG und die bisherige Rechtsprechung des Bundesrates in Tarifsachen soll nachfolgend ein kurzer Überblick über die möglichen Fälle gegeben werden

\section{Beschwerde gegen die Genehmigung eines Tarifvertrages zwischen einem oder mehreren Versicherern oder deren Ver- band einerseits und einem oder mehreren Leistungserbringern oder deren Verband andererseits durch die Kantonsregierung (Art. 46 Abs. 4 KVG)}

Nach Artikel 46 Absatz 2 KVG sind Tarifverträge für Verbandsmitglieder nur dann verbindlich, wenn sie aktiv beigetreten sind. Damit ist kein Arzt und keine Ärztin gezwungen, einem Vertrag beizutreten. Treten einzelne Verbandsmitglieder nicht bei, so können sie den Genehmigungsbeschluss nicht anfechten, da sie durch diesen nicht betroffen sind und kein schutzwürdiges Interesse nachweisen können [3]. Gleiches gilt für den Fall, dass ein Dritter die Genehmigung eines Einzelvertrages zwischen einem Versicherer und einem Leistungserbringer anfechten will. Auf derartige Beschwerden wird daher nicht eingetreten.

\section{Beschwerde gegen die Festsetzung des Taxpunktwertes durch die Kantonsregie- rung (Art. 47 Abs. 1 KVG)}

Werden sich Versicherer und Leistungserbringer im Verband oder einzeln über die Höhe des Taxpunktwertes nicht einig oder tritt jemand keinem Verbandsvertrag bei, so ist es Aufgabe der Kantonsregierung, den Taxpunktwert festzusetzen [4]. Dieser Beschluss kann von den dadurch Betroffenen grundsätzlich angefochten werden.

Treten indessen lediglich einzelne Versicherer oder Leistungserbringer als Beschwerdeführer auf und besteht ein genehmigter Vertragstarif, so stellt sich die Frage, ob die Beschwerdeführer ihrer Verhandlungspflicht in genügender Weise nachgekommen sind. Wer nämlich einer bestehenden und genehmigten Vereinbarung fernbleibt, ohne sich um eine individuelle Vereinbarung zu bemühen oder ohne eigene begründete Vorschläge zur Tarifgestaltung zu unterbreiten, muss hinnehmen, dass wegen des Vorrangs der vertraglichen Vereinbarung von Tarifen der genehmigte und damit vermutungsweise KVGkonforme - Tarifvertrag ohne weiteres als Tarif im vertragslosen Zustand festgesetzt wird. Ausnahmen von diesem Grundsatz können dann angebracht sein, wenn der Tariferlass Modalitäten enthält, die gegen zwingende Vorschriften des KVG verstossen, so dass das öffentliche Interesse an der Korrektur des angefochtenen Tariferlasses jenes an der Aufrechterhaltung der vom Gesetzgeber vorgesehenen Art und Weise des Zustandekommens von Tarifen überwiegt. In diesen Fällen wird eine materielle Überprüfung des Tariferlasses vorgenommen, auch wenn Verbandsmitglieder, welche dem von ihrem Verband abgeschlossenen Tarifvertrag nicht beigetreten sind, der geforderten Pflicht, mit der Gegenseite zu verhandeln, nicht nachgekommen sind [5]. 


\section{Beschwerde gegen die Nichtfestsetzung eines Taxpunktwertes durch die Kantonsregierung - Rechtsverweigerung}

Nicht nur das Tätigwerden, auch das Nichttätigwerden einer kantonalen Regierung kann einer Überprüfung durch den Bundesrat unterzogen werden. Wird daher - trotz Antrag - kein Taxpunktwert festgesetzt, so kann dies von den Betroffenen angefochten werden. $\mathrm{Zu}$ prüfen ist in jedem Fall das schutzwürdige Interesse. Zudem gelten die Ausführungen unter voranstehendem Punkt zur Verhandlungspflicht auch hier.

\section{Fazit}

Mit Blick auf die konstante bundesrätliche Rechtsprechung ist der Spielraum für Beschwerden gegen kantonale Taxpunktwerte auf Fälle beschränkt, in denen sich Verbände oder einzelne Partner nicht einig werden. Welches Resultat die Beurteilung der Beschwerden ergibt, hängt von den Beschwerdegründen ab.
Literatur

1 Artikel $47 \mathrm{KVG}$

2 Artikel $53 \mathrm{KVG}$

3 Artikel 48 Buchstabe a VwVG, vgl. dazu RKUV 5/2001, KV 178, S. 371ff. Wenn ein Verbandsmitglied einem Tarifvertrag nicht beigetreten ist (Art. 46 Abs. $2 \mathrm{KVG}$ ), ist es zur Beschwerde gegen dessen Genehmigung nicht legitimiert, weil es durch den Vertrag und dessen Genehmigung gar nicht beschwert ist. Umgekehrt liegt im Beitritt $\mathrm{zu}$ einem Tarifvertrag und in der Anfechtung der Genehmigung des nämlichen Vertrages ein widersprüchliches und gegen den Grundsatz von Treu und Glauben verstossendes Verhalten, mit der Konsequenz, dass der Rechtsschutz auch hier versagt bleiben muss.

4 Zur Rechtsprechung des Bundesrates: RKUV 3/2002, KV 214, S. 202 und RKUV 4/2002,

KV 220, S. 309 und KV 232, S. 480. Zu beachten ist, dass die Kantonsregierung nur den Taxpunktwert und allfällige für die Umsetzung des Tarifes notwendige Modalitäten festlegen kann, nicht jedoch den Wechsel zum System des «tiers payant» nach Artikel 42 KVG, RKUV4/2002, KV 218, S. 309.

5 RKUV 2/1999, KV 70, S. 169 sowie unpublizierter Entscheid vom 6. November 2002 zu einer Tariffestsetzung durch die Kantonsregierung.

Tabelle 1

Überblick über die Beschwerdemöglichkeiten.

\begin{tabular}{|c|c|c|}
\hline & $\begin{array}{l}\text { Beschwerdeführer/in dem } \\
\text { genehmigten Vertrag beigetreten }\end{array}$ & $\begin{array}{l}\text { Beschwerdeführer/in dem } \\
\text { genehmigten Vertrag nicht beigetreten }\end{array}$ \\
\hline $\begin{array}{l}\text { Tarifvertrag genehmigt } \\
\text { (Art. } 46 \text { Abs. } 4 \text { KVG) }\end{array}$ & Nein & Nein \\
\hline $\begin{array}{l}\text { Tariffestsetzung } \\
\text { (Art. } 47 \mathrm{KVG} \text { ) }\end{array}$ & Nein & Ja \\
\hline $\begin{array}{l}\text { Rechtsverweigerung } \\
\text { wegen Nichtfestsetzung }\end{array}$ & Nein & Ja \\
\hline
\end{tabular}

